\title{
Identification of Torque Teno Virus/Torque Teno-Like Minivirus in the Cervical Lymph Nodes of Kikuchi-Fujimoto Lymphadenitis Patients (Histiocytic Necrotizing Lymphadenitis): A Possible Key to Idiopathic Disease
}

\author{
Yosep Chong $^{a} \quad$ Ji Young Lee ${ }^{a}$ Chang Suk Kang ${ }^{a, b}$ Eun Jung Lee ${ }^{a, c}$ \\ a Department of Hospital Pathology, College of Medicine, The Catholic University of Korea, Seoul, \\ Republic of Korea; ${ }^{\mathrm{b}}$ Department of Pathology, Samkwang Medical Laboratories, Seoul, \\ Republic of Korea; ' Department of Pathology, Shinwon Medical Foundation, Gwangmyeong-si, \\ Republic of Korea
}

\begin{abstract}
What Is It about?
Kikuchi-Fujimoto disease, also known as histiocytic necrotizing lymphadenitis, is a relatively uncommon, benign lymphadenopathy that mainly involves the cervical lymph nodes of young Asian women. Its etiology remained a mystery since first described by Japanese researchers. Torque teno virus/torque teno minivirus (TTV/TTMV) was first found in a Japanese hepatitis patient. Very little is known about this virus, except that the prevalence in the serum of normal blood donors is considerably higher than would be expected, suggesting a connection to some human diseases. Many studies have provided pathogenic evidence of TTV/TTMV in human diseases such as hepatitis, idiopathic pulmonary fibrosis, etc.
\end{abstract}

\section{Keywords}

Histiocytic necrotizing lymphadenitis · Kikuchi-Fujimoto disease $\cdot$ Torque teno virus/torque teno minivirus · Lymphadenitis

\begin{abstract}
Kikuchi-Fujimoto disease (KFD) is rare, and many infectious agents have been suspected for its etiology. This report presents an interesting case of KFD found with torque teno virus/ torque teno minivirus (TTV/TTMV), which closely resembles the circovirus that causes necrotizing lymphadenitis in pigs. Three Korean patients showed several enlarged lymph nodes in their neck. Quantitative polymerase chain reaction ( $(\mathrm{PCR})$ and subsequent DNA sequencing for TTV/TTMV using formalin-fixed paraffin-embedded tissue were performed. Histologic examination demonstrated typical features of KFD. qPCR showed successful amplification of TTV/TTMV, and DNA sequencing confirmed the results. It is the first report of TTV/TTMV presence in three patients with KFD.




\section{Established Facts}

- Kikuchi-Fujimoto infection (KFD) is an uncommon disease that mostly affects Japanese and Asian people.

- Torque teno/torque teno-like minivirus (TTV/TTMV) causes necrotizing lymphadenitis in pigs, histologically similar to KFD.

\section{Novel Insights}

- $\quad$ Our study presents very interesting cases of KFD found in TTV/TTMV.

- This is the first clinical study reported on TTV/TTMV in 3 KFD patients.

\section{Introduction}

In this report, we present fascinating cases of torque teno/torque teno-like minivirus (TTV/TTMV) found in three Kikuchi-Fujimoto disease (KFD) patients, by quantitative polymerase chain reaction (qPCR) using formalin-fixed paraffin-embedded tissue of excised lymph nodes, and discuss a possible relationship between TTV/TTMV and KFD.

\section{Case Presentation}

The first patient presented with several palpable lymph nodes on the left-hand side of her neck following mild tenderness for 3 weeks [1]. The second 10-year-old male patient showed multiple lymph nodes on the left neck with central necrosis observed under ultrasonography, and the third 20-year-old female patient presented with right cervical lymph node, sore throat, dysphagia, and no fever or chill. All patients visited the local clinic, where a 1-week treatment did not regress the swelling. All patients had no special social or past history of blood transfusion and drug intake. On ultrasonography, multiple enlarged lymph nodes were found along the left posterior triangle and upper internal jugular chain (data not shown). No particular necrotic portion was noted. The initial clinical impression was tuberculosis lymphadenitis. Subsequently, an excisional biopsy of the lymph nodes was performed in each patient.

Microscopic examination revealed characteristic features of histiocytic necrotizing lymphadenitis (Fig. 1). The nodal architecture was partially maintained with occasionally opened sinuses, indicating the benign nature of this lesion. Multifocal areas of necrosis, hemorrhage, and hyalinization were noted with the diffuse replacement of histiocytes and karyorrhectic cytotoxic T lymphocytes. A preliminary diagnosis of KFD was made based on these histologic findings. The results of periodic Acid-Schiff and methenamine silver stains for the fungal organism, Warthin-Starry stain for Bartonella henselae, tissue Gram stain for bacterial organisms, Ziehl-Neelsen stain for acid-fast bacilli, PCR for tuberculosis, and in situ hybridization for Epstein-Barr virus were all negative. After lymph node ablation, the patient was treated conservatively, and the remaining enlarged cervical lymph nodes slowly regressed.

DNA was extracted from the paraffin-embedded tissue block using Gentra ${ }^{\circledR}$ Puregene ${ }^{\circledR}$ Tissue kit. The extracted DNA sample was purified using a MinElute ${ }^{\circledR}$ purification kit. The 

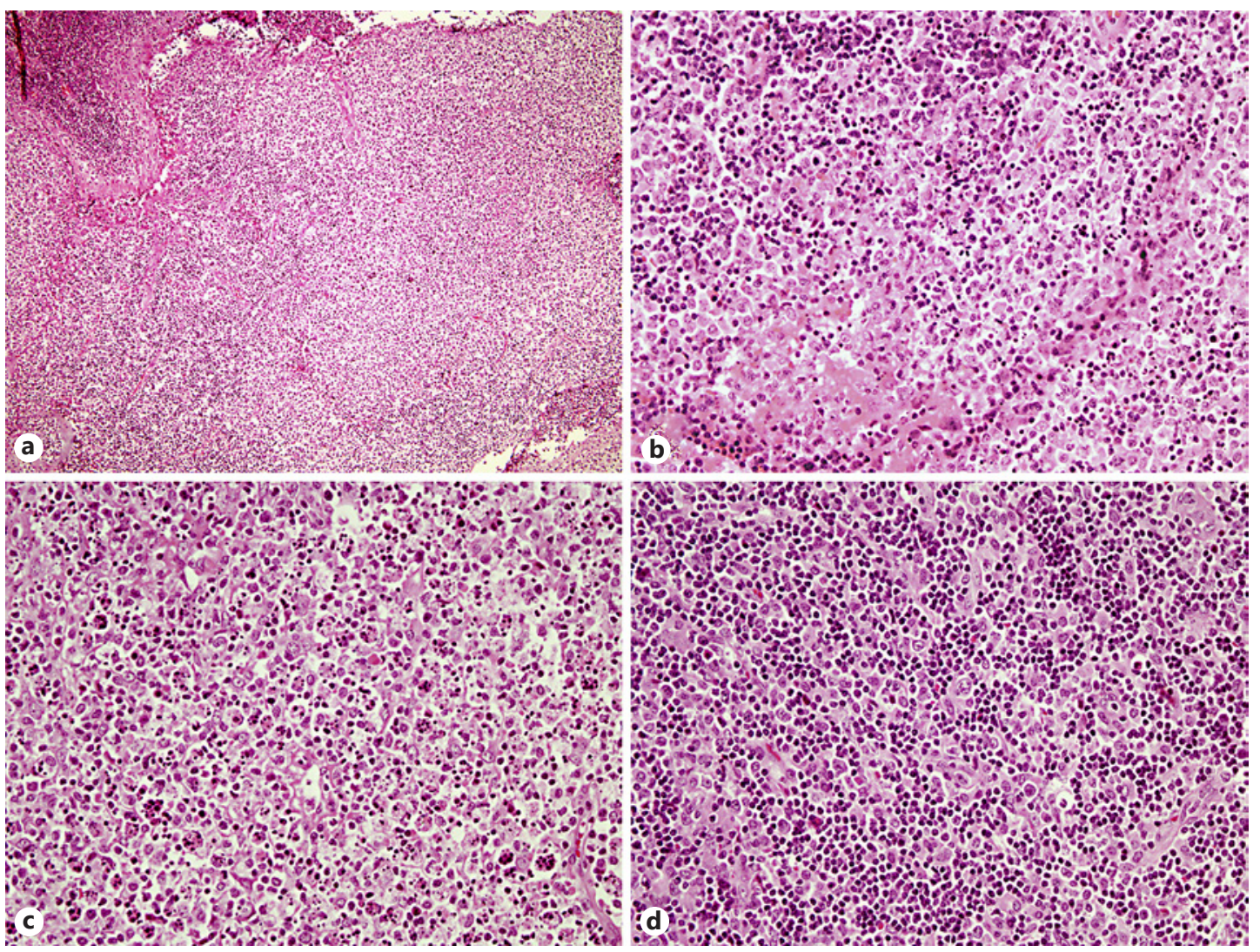

Fig. 1. Microscopic findings. Low-power magnification reveals partial effacement of nodal architecture by suppurative inflammation with vague necrosis (a). High magnification reveals extensive inflammatory cell infiltration in the karyorrhectic background (b), area of numerous phagocytic histiocytes (c), and area of lymphocytic proliferation (d).

purified DNA was quantified using Nanodrop ${ }^{\circledR} 1000$ spectrophotometer. qPCR amplification was performed as previously described [2]: TTV/TTMV-F; 5'-TTC GAA TGG CTG AGT TT-3' (102-118), and TTV/TTMV-R; 5'-CGA ATT GCC CCT TGA CT-3' (219-203). The PCR amplification by gel electrophoresis confirms the band at $96 \mathrm{bp}$. The Ct value of the three patients were 34.6, 37.4, and 36.9 for TTV, and 30.9, 30.8, and 31.1 for TTMV. Subsequent DNA sequencing with forward and reverse primer was done for TTV, TTMV, and obtained sequences were searched in the BLAST databases (https://blast.ncbi.nlm.nih.gov/Blast.cgi).

\section{Discussion}

This first report of TTV/TTMV presence in three patients with KFD has two important implications. First, these cases present a possible pathogenic role for TTV/TTMV in KFD. Second, it calls attention to the requirement for further exploration into the potential pathogenicity of TTV/TTMV in human diseases.

This case implies a potential role for TTV/TTMV in the pathogenesis of KFD. Despite the relatively well-established clinicopathologic features of KFD, the etiology of this disease has remained unclear for more than 40 years since its first recognition [3]. A diagnosis of KFD can only be made by a process of exclusion of all other causes of lymphadenitis; this process is 
vital as KFD can present in a very similar way to other lymphoid malignancies, but the treatment is markedly different $[4,5]$. It can be time-consuming and sometimes problematic in an outpatient clinic in countries where the prevalence of KFD is relatively high, such as Korea or Japan [5]. The absence of targeted treatment is another critical reason for the cause of this disease to be identified. In our previous meta-analysis, we evaluated many infectious human pathogenic viruses and bacteria, but none of them were found to be associated with KFD [6]. For this reason, TTV/TTMV found in the excised lymph node tissue is a remarkable finding. Undoubtedly, further investigation is needed to support this finding.

The findings of the present cases also potentially implicate TTV/TTMV in the pathogenicity of other human diseases. After its first discovery by Nishizawa et al. [7] in a Japanese patient with non-A to G post-transfusion hepatitis, the possibility of the involvement of TTV/ TTMV in the pathogenicity of some human diseases has been raised [7, 8]. However, subsequent studies have indicated a high "carrier" state in chronic hepatitis patients, and even in normal blood donors $[9,10]$. Although a few studies have suggested a possible pathogenic role for TTV/TTMV in diseases such as idiopathic pulmonary fibrosis, whether a high prevalence in various test samples in normal hosts, e.g., saliva, feces, and serum, indicates a chronic persistent or latent infection has not been clearly elucidated [10-12]. However, the identification of TTV/TTMV in tissue samples involving definite pathologic processes, such as necrotizing lymphadenitis in the present case, is a novel finding that should drive further investigation into the pathogenic role of TTV/TTMV in human disease.

Further interest is added to this case when the classification of TTV/TTMV is considered. Initially, it classified as a member of the Circoviridae family, which includes the circovirus that causes necrotizing lymphadenitis in pigs [13]. Circovirus is structurally similar to TTV/TTMV; it is a single-stranded circular DNA virus without an envelope and mainly infects mammals and can cause fever, neutropenia, weight loss, and granulomatous inflammation in postweaning multisystemic wasting syndrome (PMWS) [13]. Recently, TTV/TTMV has been reclassified into a new family, Anelloviridae. However, the pathologic features shared by KFD in humans and necrotizing lymphadenitis of PMWS in pigs should not be overlooked [10].

We did not find any unusual clinicopathologic findings to differentiate from typical KFD in these cases. More studies with larger numbers of cases are required for the detail information of the clinicopathologic feature of KFD with TTV/TTMV.

\section{Acknowledgements}

I appreciate Seung Bum Hong for DNA sequencing. I thank Young Dong Seo and Nishant Thakur for reviewing the manuscript style.

\section{Statement of Ethics}

This study was reviewed and approved by the Institutional Review Board of The Catholic University of Korea College of Medicine (SC14TISI0016) with a waiver of consent.

\section{Disclosure Statement}

The authors have no conflicts of interest to declare. 
Chong et al.: Three KFD Patients with TTV/TTMV

\section{Funding Sources}

This study was partly funded by a research grant from the Institute of Clinical Medicine Research, Catholic University of Korea, Yeouido St. Mary's Hospital.

\section{Author Contributions}

Y.C. and C.S.K. designed the study, C.S.K. and E.J.L. supervised the study, Y.C. and J.Y.L. performed the experiments and data curation, Y.C. wrote the draft, and C.S.K. and E.J.L. reviewed the manuscript. All authors read and approved the report.

\section{References}

1 Chong Y, Lee JY, Thakur N, Kang CS, Lee EJ. Strong association of Torque teno virus/Torque teno-like minivirus to Kikuchi-Fujimoto lymphadenitis (histiocytic necrotizing lymphadenitis) on quantitative analysis. Clin Rheumatol. 2019 Nov. DOI: 10.1007/s10067-019-04851-4.

2 Moen EM, Sleboda J, Grinde B. Real-time PCR methods for independent quantitation of TTV and TLMV. J Virol Methods. 2002 Jun;104(1):59-67.

3 Bosch X, Guilabert A, Miquel R, Campo E. Enigmatic Kikuchi-Fujimoto disease: a comprehensive review. Am J Clin Pathol. 2004 Jul;122(1):141-52.

4 Ioachim HL, Medeiros LJ. Ioachim's lymph node pathology. 4th ed. Philadelphia: Wolters Kluwer/Lippincott Williams \& Wilkins; 2009.

5 Han KM, Go JH, Myong NH, Lee W. Fine Needle Aspiration Cytology of Kikuchi's Lymphadenitis: with Emphasis on Differential Diagnosis with Tuberculosis. Korean J Pathol. 2011 Dec;45(6):626-31.

6 Chong Y, Kang CS. Causative agents of Kikuchi-Fujimoto disease (histiocytic necrotizing lymphadenitis): a meta-analysis. Int J Pediatr Otorhinolaryngol. 2014 Nov;78(11):1890-7.

7 Nishizawa T, Okamoto H, Konishi K, Yoshizawa H, Miyakawa Y, Mayumi M. A novel DNA virus (TTV) associated with elevated transaminase levels in posttransfusion hepatitis of unknown etiology. Biochem Biophys Res Commun. 1997 Dec;241(1):92-7.

8 Cossart Y. TTV - a virus searching for a disease. J Clin Virol. 2000 Jun;17(1):1-3.

9 Naoumov NV, Petrova EP, Thomas MG, Williams R. Presence of a newly described human DNA virus (TTV) in patients with liver disease. Lancet. 1998 Jul;352(9123):195-7.

10 Hino S, Miyata H. Torque teno virus (TTV): current status. Rev Med Virol. 2007 Jan-Feb;17(1):45-57.

11 Thom K, Morrison C, Lewis JC, Simmonds P. Distribution of TT virus (TTV), TTV-like minivirus, and related viruses in humans and nonhuman primates. Virology. 2003 Feb;306(2):324-33.

12 Bando M, Takahashi M, Ohno S, Hosono T, Hironaka M, Okamoto H, et al. Torque teno virus DNA titre elevated in idiopathic pulmonary fibrosis with primary lung cancer. Respirology. 2008 Mar;13(2):263-9.

13 Chae C. A review of porcine circovirus 2-associated syndromes and diseases. Vet J. 2005 May;169(3):326-36. 